\title{
Parapneumonic Effusion and Tension Pneumothorax after Diverticular Peroral Endoscopic Myotomy in a Woman with Large Epiphrenic Diverticulum: A Case Report and Literature Review
}

\author{
Sz-luan Shiu' ${ }^{1,2,3}$ \\ ${ }^{1}$ Division of Gastroenterology and Hepatology, Department of Internal Medicine, ${ }^{2}$ Department of Critical Care Medicine, ${ }^{3}$ Evidence- \\ based Practice and Policymaking Committee, Taichung Veterans General Hospital, Taichung, Taiwan
}

Esophageal diverticula (ED) represents a group of rare conditions that warrant intervention when they are symptomatic or coexisting with pulmonary disorders. Few literature reviews have described this patient entity or discussed the postoperative outcome. Therefore, I present the case of a 59-year-old woman with symptoms of dysphagia who was significantly underweight, which was conducive to the diagnosis of symptomatic ED. Because she was a poor candidate for surgery, she received a diverticular peroral endoscopic myotomy. She subsequently developed parapneumonic effusion and tension pneumothorax after the procedure. She was finally discharged on postoperative day 23. I also performed the first known comprehensive literature review of 34 published cases (including my patient) from PubMed and have addressed the demography, intervention, and prognosis for symptomatic ED after the procedure. Prompt treatment as well as prognostic measurement are crucial to successful outcomes. Clin Endosc 2021;54:275-279

Key Words: Epiphrenic diverticulum; Parapneumonic effusion; Peroral endoscopic myotomy; Tension pneumothorax

\section{INTRODUCTION}

Esophageal diverticula (ED) involves pulsion of the mucosa and submucosa herniating through the muscularis propria in the esophagus, ${ }^{1}$ with a rare prevalence of between $0.22 \%$ to $2 \%$, as seen in epidemiological studies. ${ }^{2}$ Non-specific esophageal motility disorder, diffuse esophageal spasm, and achalasia commonly coincide with epiphrenic ED if the condition involves the distal esophagus, while thoracic ED is often associated with diffuse motility disorder. ${ }^{2,3}$ Symptomatic $\mathrm{ED}$ or high risk of aspiration pneumonia due to large ED may

Received: April 7, 2020 Revised: May 23, 2020

Accepted: May 25, 2020

Correspondence: Sz-Iuan Shiu

Division of Gastroenterology and Hepatology, Department of Internal Medicine, Taichung Veterans General Hospital, No. 1650 Taiwan Boulevard Sect. 4, Taichung 40705, Taiwan

Tel: +886-4-2359-2525, Fax: +886-4-2374-1331, E-mail: b9002007@hotmail.com ORCID: https://orcid.org/0000-0003-4385-5095

(c) This is an Open Access article distributed under the terms of the Creative Commons Attribution Non-Commercial License (http://creativecommons.org/ licenses/by-nc/3.0) which permits unrestricted non-commercial use, distribution, and reproduction in any medium, provided the original work is properly cited. provide grounds for intervention, ${ }^{4,5}$ which could be managed surgically. Possible procedures include open versus laparoscopic, diverticulectomy versus diverticulopexy, routine versus selective myotomy, and selective anti-reflux fundoplication. ${ }^{6,7}$ Alternative endoscopic treatments involving traditional peroral endoscopic myotomy (POEM), salvage POEM (S-POEM), and diverticular POEM (D-POEM) have been recently introduced for symptomatically poor candidates for surgery. ${ }^{8}$ Traditional POEM entails performing a submucosal endoscopic myotomy on the inner layer of the esophagus at the 6 or 12 oclock position, while S-POEM involves myotomy surgery on the wall opposite the ED. ${ }^{9}$ D-POEM requires septotomy after creating submucosal tunneling twice on both sides of the ED. The demography, optimal management, and prognosis are yet unclear in patients with symptomatic ED. I present a case of large ED in a woman that was complicated by parapneumonic effusion and tension pneumothorax after D-POEM; I also analyze the demography, intervention, and prognosis from a systemic literature review. 


\section{CASE REPORT}

The patient was a 59-year-old woman who presented to the outpatient clinic with gradual-onset dysphagia that had developed over 2 years. She also reported acid regurgitation, retrosternal pain, nausea, vomiting, poor appetite, and significant weight loss of approximately 18 kilograms, all occurring simultaneously in the past six months. She had a medical history of severe asthma, associated with steroid-induced diabetes mellitus and required stepwise combination therapy involving an inhaled corticosteroid plus a long-acting beta-agonist. However, owing to multiple drug allergies, she could only receive prednisolone $20 \mathrm{mg}(0.5 \mathrm{mg} / \mathrm{kg} /$ day $)$ daily and inhaled salbutamol nebule four times a day to minimize acute exacerbation. Initially she was diagnosed as having epiphrenic ED owing to hematemesis occurring 2 years prior to admission and becoming symptomatic over the past six months. She received a percutaneous endoscopic gastrostomy 3 months prior to admission, but still suffered from recurrent aspiration pneumonia due to subsequently spilling saliva from ED. Upon physical examination at admission, her body mass index was $17.5 \mathrm{~kg} / \mathrm{m}^{2}$. A chest examination revealed diffuse wheezing bilaterally during auscultation, while the rest of the physical examination was unremarkable. A laboratory examination showed a total leukocyte count of 13,470 cells per $\mu \mathrm{L}$, hemoglobin concentration of $13.8 \mathrm{~g} / \mathrm{dL}$, and platelet count of 328,000 per $\mu \mathrm{L}$ upon initial presentation. A chest radiograph showed an outpouching sac of $8 \times 6 \mathrm{~cm}$ diameter with an air-fluid level in the distal $10 \mathrm{~cm}$ of the esophagus. Additionally, barium esophagography plus computed tomography (CT) of the chest exhibited comparative results (Fig. 1A, B). An upper endoscopy revealed large epiphrenic ED visualized in the distal esophagus approximately $32 \mathrm{~cm}$ from the incisors, while the remaining distal esophagus was so narrow that only a pediatric endoscope could pass through (Fig. 2A). Esophageal manometry failed to pass the probe through the distal esophagus.

After the patient was admitted to the general ward, I performed D-POEM with endoscopic myotomy $3 \mathrm{~cm}$ beyond the distal end of ED in addition to a diverticuloseptotomy, which was performed with the patient in the left decubitus position under general anesthesia using endotracheal intubation and carbon dioxide insufflation. An endoscope with a short STHood (DH-28GR; Fujifilm, Tokyo, Japan) and Triangle-Tip Knife (KD-640L; Olympus, Tokyo, Japan) was used during the whole procedure. A mucosotomy was created $29 \mathrm{~cm}$ from the incisors, followed by submucosal tunneling between the diverticular mucosa and the diverticular septum (Fig. 2B, C). A parallel tunnel was then created within the submucosa of the true esophageal lumen, with an extension to $38 \mathrm{~cm}$ from the incisors. This however did not pass through the lower esophageal sphincter due to sharply arising tunnel pressure, as confirmed using a double pediatric scope. The complete muscle layer of the diverticular septum was then dissected to the bottom of the diverticulum and further myotomy was performed by dividing the circular muscle layer of the distal esophagus $3 \mathrm{~cm}$ beyond the distal end of ED (Fig. 2D-F). Subcutaneous emphysema occurred on the right side of the neck and chest immediately after D-POEM, without any unstable vital signs being seen (Fig. 3A). However, exertional dyspnea with left pleuritic pain developed on postoperative day 3; a chest CT showed massive pleural effusion and pulmonary atelectasis of the left side (Fig. 3B), indicating uncomplicated parapneumonic effusion via thoracentesis. A chest tube was immediately inserted with maintenance using negative pressure, but a follow-up esophagoscopy uncovered a spontaneous
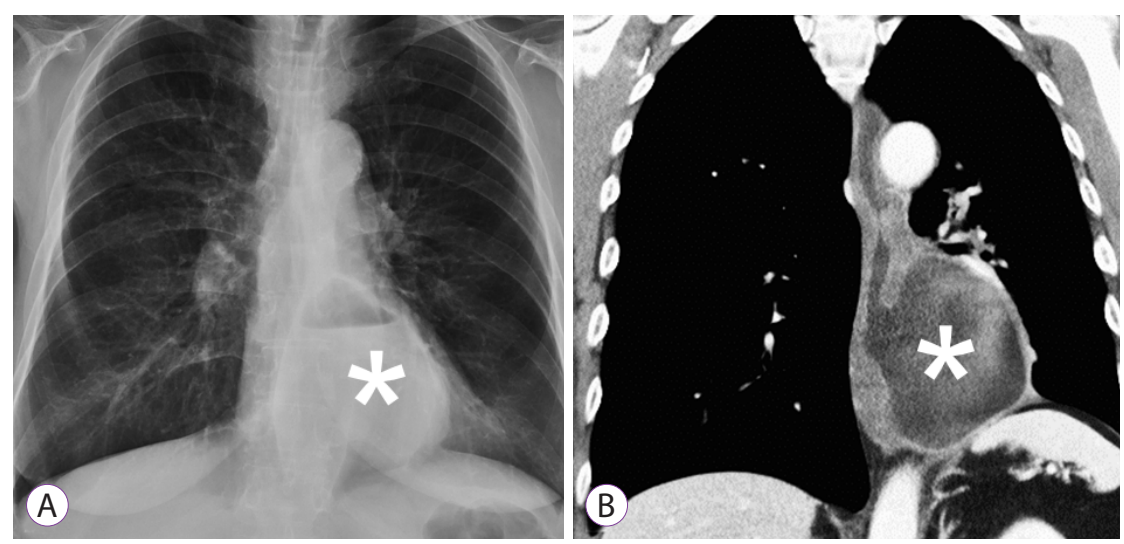

Fig. 1. An esophageal diverticulum. (A) Chest radiograph shows an esophageal diverticulum (asterisk) measuring $80 \times 60 \mathrm{~mm}$. (B) Computed tomography of the chest shows an outpouching sac with air-fluid levels in the distal $10 \mathrm{~cm}$ of the esophagus. 

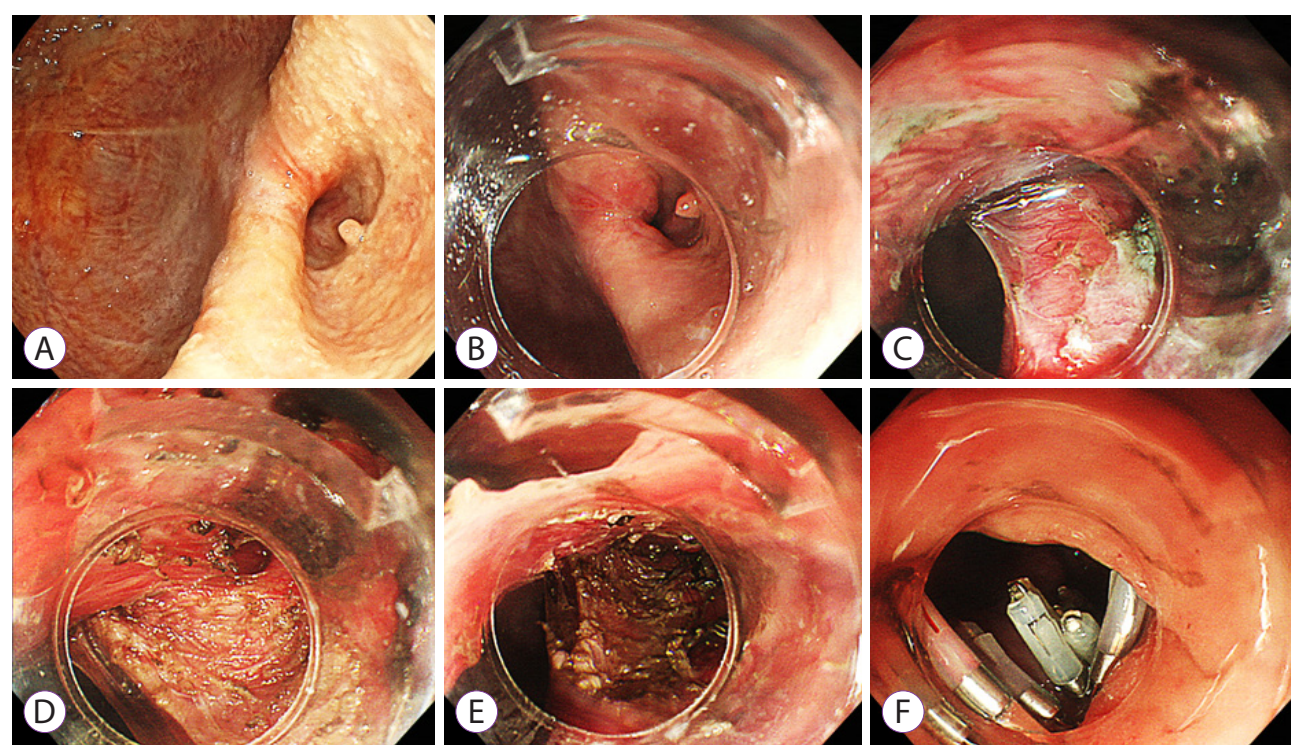

Fig. 2. (A) Upper endoscopy showed a large epiphrenic esophageal diverticulum and narrowed lumen at the distal esophagus. A diverticular peroral endoscopic myotomy was performed stepwise as follows: (B) mucosotomy was created $3 \mathrm{~cm}$ from the diverticular septum, (C) submucosal tunneling was followed, (D) a diverticuloseptotomy was performed to the bottom of diverticulum, (E) further myotomy was performed by dividing the circular muscle layer of the distal esophagus 3 $\mathrm{cm}$ beyond, and (F) endoclips were applied for final closure.
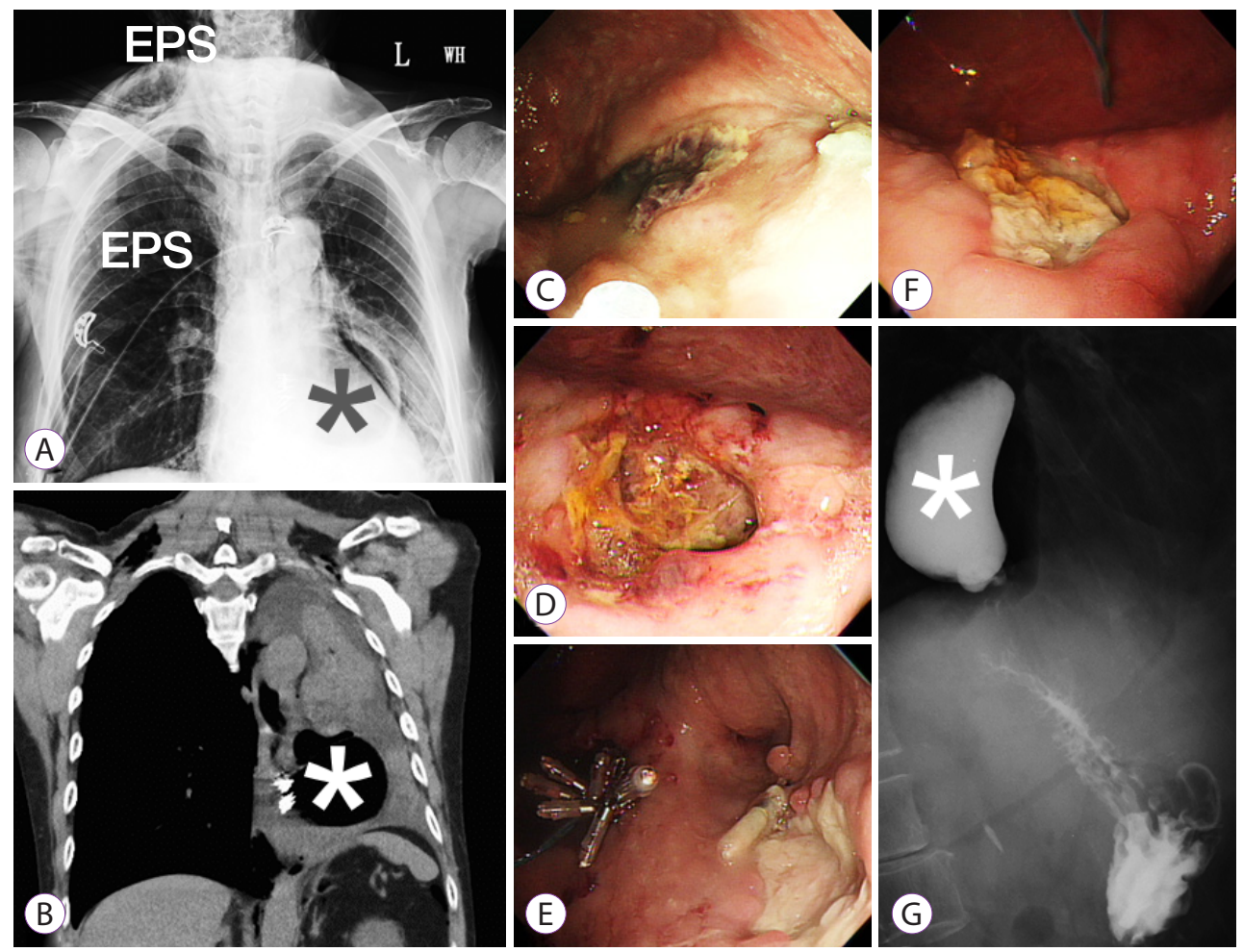

Fig. 3. (A) Subcutaneous emphysema (EPS) was noted on the neck and chest of the right side after the procedure. (B) On postoperative day 3 , a computed tomography of the chest showed massive pleural effusion and pulmonary atelectasis of the left side. (C) A follow-up esophagoscopy showed a spontaneous mucosal defect within the esophageal diverticulum on postoperative day 5. (D) On postoperative day 11, esophagoscopy showed an enlarged esophageal ulcer with suspicious communication to the pleural space. (E) Mucosal closure with an endoloop and endoclips was applied. (F) On postoperative day 16, esophagoscopy revelaed a healing ulcer. (G) A barium esophagography showed smooth passage of barium to the gastric lumen without any additional leakage on postoperative day 22. Asterisk indicates an esophageal diverticulum. 
mucosal defect within ED on postoperative day 5 (Fig. 3C). I applied endoclips for complete closure of the mucosal defect. The following day tension pneumothorax was noted via a follow-up chest radiograph and a second follow-up esophagoscopy on postoperative day 11 was performed due to continuous pneumothorax under maintenance of negative pressure via a chest tube. This manifested an enlarged mucosal defect with suspicious communication to the pleural space; therefore, mucosal closure utilizing an endoloop and endoclips was thoroughly applied (Fig. 3D, E). On postoperative day 16, a third follow-up esophagoscopy revealed a healing ulcer as well as resolution of the pneumothorax; the chest tube was removed the following day (Fig. 3F). A barium esophagography showed the decreasing size of ED and a smooth passage to the gastric lumen without any additional leakage on postoperative day 22 (Fig. 3G). The patient attempted the intake of water and a clear liquid diet on postoperative day 17 after removal of the chest tube and has since tolerated a liquid diet. I prescribed antibiotics Unasyn 3 g every 12 hours for a total of 22 days from postoperative day 1 for subcutaneous emphysema immediately after D-POEM and discontinued the medication one day prior to discharge. The patient was then discharged on postoperative day 23 in a stable condition.

\section{DISCUSSION}

Symptomatic ED are rare but challenging in clinical practice, with a total of 34 cases (including the present case) being reported in the literature (Supplementary Table 1). The mean patient age at admission is approximately 71 years, with a female predominance $(62 \%)$. Symptomatic ED often presents with dysphagia (100\%), chest pain (38\%), acid regurgitation (31\%), and weight loss (16\%), in order of occurrence. As seen in my review, the mean duration after onset of symptoms was 8 years, patients with ED experienced achalasia in more than half the cases, and the etiology was idiopathic in approximately one third of cases.

In my study, the overall complication rate was $21 \%$, with the use of endoscopic intervention POEM alone being performed in $61 \%$ patients, while combined treatments with D-POEM, D-POEM alone, and S-POEM alone were performed in 12\%, $21 \%$, and $6 \%$ patients, respectively. Adverse events after disease management included pneumoperitoneum, left pleural effusion/parapneumonic effusion, subcutaneous emphysema, or tension pneumothorax, particularly in patients who presented with weight loss and lesions in the distal esophagus.

The epidemiology and prognosis in a population with symptomatic ED, or in those with a high risk of aspiration pneumonia prompting for early intervention, remains elusive. One retrospective cohort study ${ }^{10}$ enrolling epiphrenic ED patients with esophageal motility disorder reported that POEM alone appeared to be a secure and effective treatment for elderly patients. Another systemic review ${ }^{7}$ involving surgical intervention for individuals with non-Zenker's ED found that the median age was 62 years, with patients experiencing epiphrenic ED predominance and a strong association with esophageal motility disorders, which showed a distribution comparative to that seen in my patients. Although the causal relationship between esophageal motility disorders and symptomatic ED is not clear, minimal invasive surgery involving diverticulectomy, myotomy, and elective fundoplication is routinely conducted. ${ }^{4,5,10,11}$ The most recent meta-analysis ${ }^{7}$ reported an in-hospital mortality rate of $5.9 \%$, pooled morbidity rate of $21.1 \%$, overall treatment success rate of $88.5 \%$, and an overall staple line leakage rate of $13.3 \%$. A diverticulectomy, in addition to surgery, is known to result in better treatment success and symptomatic resolution, while staple line leakage could be reduced by performing routine myotomy. No consensus is available regarding the surgical procedure of ED in reducing patient preference due to a higher postoperative morbidity rate.

POEM was initially utilized in Zenker's diverticulum ${ }^{12,13}$ and has recently been performed successfully in non-Zenker's diverticulum by implementing alternative endoscopic techniques involving traditional POEM, S-POEM, and D-POEM. However, there is still no consensus regarding the priority of their use. When patients are experiencing symptomatic ED and achalasia simultaneously, either traditional POEM or S-POEM could be initially considered, depending upon individual experience, team familiarity with endoscopic performance, and complexity of the intertwined muscles fibers from the ED to the gastroesophageal junction. When an endoscopist decides to perform D-POEM, the choice of whether to conduct additional myotomy on the inner muscle beyond the diverticulum depends upon the underlying motility disorders, and whether there is enough submucosal space for an endoscope to pass through. In two of the latest retrospective cohort studies ${ }^{8,10}$ commended D-POEM was found to be relatively safe in an averaged ED diameter of 29 (9-90) $\mathrm{mm}$ and 34.5 (10-53) mm separately, but the treatment lacks further evidence surrounding the safety when managing larger ED. My patient developed parapneumonic effusion and tension pneumothorax after D-POEM, which may have originated from a spontaneous esophageal rupture or delayed perforation, owing to thermal injury via electrocautery. Sato et al. reported that epiphrenic ED were more likely to occur at the right esophageal wall ${ }^{13}$ and tended to rupture spontaneously through the 
left wall. It should be noted that diverticular mucosa becomes thinner when the size of ED is enlarged, which may also be harmful to the patient. This is in addition to there also being certain risk factors, including older age, multiple comorbidities, or being underweight.

In conclusion, this is, to the best of my knowledge, the first comprehensive literature review relative to symptomatic ED that discusses the demography, intervention, and prognosis after procedure. This review emphasizes the demand for comprehensive evaluation and personalized treatment to achieve a beneficial postoperative outcome. Further studies are still warranted regarding the safety of endoscopic myotomy.

Conflicts of Interest

The author has no potential conflicts of interest.

Funding

None.

Acknowledgments

The Evidence-based Practice and Policymaking Committee, Taichung Veterans General Hospital, Taichung, Taiwan, is acknowledged.

Author Contributions

Conceptualization: Sz-Iuan Shiu

Data curation: SIS

Formal analysis: SIS

Investigation: SIS

Methodology: SIS

Project administration: SIS

Writing-original draft: SIS

Writing-review\&editing: SIS

\section{ORCID}

Sz-Iuan Shiu:

https://orcid.org/0000-0003-4385-5095

\section{REFERENCES}

1. Soares R, Herbella FA, Prachand VN, Ferguson MK, Patti MG. Epiphrenic diverticulum of the esophagus. From pathophysiology to treatment. J Gastrointest Surg 2010;14:2009-2015.

2. Thomas ML, Anthony AA, Fosh BG, Finch JG, Maddern GJ. Oesophageal diverticula. Br J Surg 2001;88:629-642.

3. Hoghooghi D, Coakley FV, Breiman RS, Qayyum A, Yeh BM. Frequency and etiology of midesophageal diverticula at barium esophagography. Clin Imaging 2006;30:245-247.

4. Fisichella PM, Jalilvand A, Dobrowolsky A. Achalasia and epiphrenic diverticulum. World J Surg 2015;39:1614-1619.

5. Herbella FAM, Patti MG. Achalasia and epiphrenic diverticulum. World J Surg 2015;39:1620-1624.

6. Eubanks TR, Pellegrini CA. Minimally invasive treatment of esophageal diverticula. Semin Thorac Cardiovasc Surg 1999;11:363-367.

7. Chan DSY, Foliaki A, Lewis WG, Clark GWB, Blackshaw G. Systematic review and meta-analysis of surgicaltreatment of non-Zenker's oesophageal diverticula. J Gastrointest Surg 2017;21:1067-1075.

8. Yang J, Zeng X, Yuan X, et al. An international study on the use of peroral endoscopic myotomy (POEM) in the management of esophageal diverticula: the first multicenter D-POEM experience. Endoscopy 2019;51:346-349.

9. Bhalla S, Reddy CA, Watts L, Chang AC, Law R. Greater-curvature peroral endoscopic myotomy with diverticuloseptotomy for the treatment of achalasia in a patient with a large epiphrenic diverticulum. VideoGIE 2020;5:77-79.

10. Kinoshita M, Tanaka S, Kawara F, et al. Peroral endoscopic myotomy alone is effective for esophageal motility disorders and esophageal epiphrenic diverticulum: a retrospective single-center study. Surg Endosc 2020;34:5447-5454.

11. Fernando HC, Luketich JD, Samphire J, et al. Minimally invasive operation for esophageal diverticula. Ann Thorac Surg 2005;80:2076-2080.

12. Manolakis AC, Inoue H, Ueno A, Shimamura Y. 2007-2019: a "Third"space odyssey in the endoscopic management of gastrointestinal tract diseases. Curr Treat Options Gastroenterol 2019;17:202-220.

13. Sato H, Takeuchi M, Hashimoto S, et al. Esophageal diverticulum: new perspectives in the era of minimally invasive endoscopic treatment. World J Gastroenterol 2019;25:1457-1464. 